\title{
Evaluating Effectiveness in Bonded Repair Techniques for Aircraft Structures using Finite Element Simulations
}

\author{
Qida Bing ${ }^{1}$, Xingshi Wang ${ }^{2}$, and Lucy Zhang ${ }^{3}$ \\ ${ }^{1}$ ANSYS Inc \\ ${ }^{2}$ BWNG LLC. \\ ${ }^{3}$ Rensselaer Polytechnic Institute
}

August 10, 2020

\begin{abstract}
Finite element simulations of bonded repair technology can greatly reduce the cost of repairing ageing and damaged aircraft structures. In this study, finite element simulation and analysis are performed for several bonded repair techniques of damaged aircraft structures with cracks. The simulations start from fatigue damage accumulation, crack initiation, crack repair, to fatigue crack re-initiation until structural failure. The effectiveness of bonded repair techniques is assessed by comparing the service lives of no repair, patch-bonded repair (live repair), stop-drill repair, and damage removal repair. It is found that the load attraction by repair patch can greatly sustain fatigue crack growth, leading to more than at least 2 times longer service life before the skin structure needs to be replaced. Damage removal bonded repair can further extend service life by more than 20 times comparing to no repair, benefiting from the fatigue damage tolerant service life extension. Along with the service life comparison, we also established a simulation framework that lays out the groundwork to perform aerostructure bonded repair effectiveness evaluation. The results demonstrate that finite element analysis can be efficiently used to simulate the various forms of bonded repairs and effectively evaluate fatigue crack growth and service life with structural damage. Such a rigorous simulation framework enables the future design of new repair techniques for aircraft structures.
\end{abstract}

\begin{abstract}
Finite element simulations of bonded repair technology can greatly reduce the cost of repairing ageing and damaged aircraft structures. In this study, finite element simulation and analysis are performed for several bonded repair techniques of damaged aircraft structures with cracks. The simulations start from fatigue damage accumulation, crack initiation, crack repair, to fatigue crack re-initiation until structural failure. The effectiveness of bonded repair techniques is assessed by comparing the service lives of no repair, patchbonded repair (live repair), stop-drill repair, and damage removal repair. It is found that the load attraction by repair patch can greatly sustain fatigue crack growth, leading to more than at least 2 times longer service life before the skin structure needs to be replaced. Damage removal bonded repair can further extend service life by more than 20 times comparing to no repair, benefiting from the fatigue damage tolerant service life extension. Along with the service life comparison, we also established a simulation framework that lays out the groundwork to perform aerostructure bonded repair effectiveness evaluation. The results demonstrate that finite element analysis can be efficiently used to simulate the various forms of bonded repairs and effectively evaluate fatigue crack growth and service life with structural damage. Such a rigorous simulation framework enables the future design of new repair techniques for aircraft structures.
\end{abstract}

Keyword : aircraft structures, finite element simulations, bonded repair, fatigue crack growth, surface crack, service life

\section{Introduction}


Bonded repair technology is an essential and vital component in aging military and commercial aircrafts [1]-[3]. As aircraft structures age, they can become progressively more susceptible to fatigue cracking and other forms of structural damage, thus can significantly impact their service life [4]. Bonded repairs can economically repair aging and damaged aircraft structures, often without removing components from the aircraft. Externally bonded composite patches are an effective method of repairing cracked or damaged structural components [5]. The bonded repair methodology was first used to repair cracks in military aircraft, it has recently been applied to civilian aircraft [6]-[9]. However, based on the Delegated Engineering authority (DEA), currently conducted repairs are heavily based on historical, experimental data (in accordance with T.O. 1-1A-81 [10] and T.O. 1-1-6912 [11]), which lacks a process that allows repeatability and further hinders the developments of new bonded repair techniques. Bonded repairs can involve bolted patch repair, adhesive bonded patch repair [12], scarf, and etc. A simulation study has shown that bolted patch repair and adhesive bonded patch repair can have different efficiencies and effectiveness [13].

Baker and Jones [1] enumerated that the conventional approach to through-life-support for aircraft structures can be divided into the following phases: (i) detection of defects, such as cracks and damage, (ii) diagnosis of their nature and significance, (iii) forecasting future behavior-prognosis, and (iv) prescription and implementation of remedial measures including repairs. Considerable scientific efforts have been devoted to the development of science and technology for the first three phases. Among them, analytical analysis of fracture mechanics in predicting residual strength in the presence of cracks (damage tolerance) and rate of crack propagation under service loading has been a major focus. Intensive effort is currently being devoted to developing similar approaches for fiber composite structures, to assess damage tolerance and durability in the presence of delamination damage [14]. Until recently there has been limited attempts to develop a process for the last phase, with respect to the evaluation of repairs. Most of the analysis tools have been focused on empirical relationships between crack growth and stress concentration factor [15]. Finite element modeling and simulation have shown promise in analyzing behaviors of aircraft structure repairs [9], [16]. However, rigorous approaches are required to allow assessment of the type and magnitude of defects amenable to repair and the influence of the repair on the stress intensity factor and most importantly the extension on their service life [17]. Such an approach is also required for the development and design of optimum repairs and for assessment of their durability.

This paper attempts to design and develop a simulation approach to address the above-mentioned technical challenges by setting up simulation framework using FEA software, Ansys [18]. Although Ansys has capabilities to perform stress analysis and even predict crack development [19], the process of correlating stress field, damage, crack initiation, crack propagation and ultimately service life estimation, is not straight-forward and requires further exploration and development of a repeatable and deployable analysis process for bonded repair applications in Damage Tolerance Analysis (DTA) on aircraft structures. Therefore, there is a critical need [10] to design and develop capabilities and a workflow that allow repeatable process where finite element analysis tools/packages, can be effectively and efficiently deployed to determine the fatigue characteristics on aircraft structures prior to, and after bonded repairs. The development of such methodology is to enable a robust and repeatable FEA engineering process so that: (i) the Bonded Repair Center of Excellence (COE) can more effectively evaluate the quality of both common fatigue driven wing plank repairs on aircraft and eventually unconventional repairs on weapon systems, (ii) the COE will be better equipped to develop a vast array of repairs with a higher degree of confidence and accuracy, (iii) the COE will be more confident in establishing maintenance service schedule.

In this study, aspects of the design process as well as the results of a constant-amplitude fatigue test program are outlined. The results of a three-dimensional finite element analysis, of both the repaired (of varying techniques) and unrepaired specimens, are presented with predictions of crack-growth rates and service life. Our developed framework can generate common and unconventional damage scenarios. To successfully develop a simulation workflow of bonded repair, we intend to address the following objectives in this study:

1. Develop a proof-of-concept "Bonded Repair Simulation Workflow" (BRSW) with Ansys suite, which carries out bonded repair design process using Finite Element (FEA)-based Damage Tolerance Analysis 
(DTA). This FEA process allows users to effectively and repeatedly evaluate bolted bonded repair design effectiveness by employing various tools offered by the existing Ansys software suite. The solution addresses the unique challenges in the bonded repair DTA analysis with advanced capabilities which are based on fatigue and fracture mechanics theories [18].

2. Validate BRSW with use-cases on predicting fatigue damage evolution, crack initiation and propagation with service life estimation, and bonded repair effectiveness. This study presents a bolted patch repair use-case, where the implemented BRSW FEA repair process can effectively quantify the damage, crack initiation, fatigue crack propagation, service life and repair effectiveness in repaired damaged aircraft structure. The BRSW process will be applied to repaired and un-repaired cracked aircraft skin to evaluate and compare their remaining service life. We first perform a COE "standard" repairing technique, namely bolted bonded patching, using BRSW process. Patch design with Titanium material is considered. The planned case studies for bolted bonded repair technique will be on a cracked pressurized fuselage structure [20].

This paper is organized as follows: in Section 2, we will introduce our proposed FEA solution workflow for aircraft damage tolerant repair using Ansys. In Section 3, we will present the case studies for a bolted bonded repair with crack initiation, fatigue crack propagation, and service life quantification with and without crack repairs. Specifically, three crack repair techniques will be examined, live crack repair, stop-drill crack repair, and damage-removal crack repair. Finally, conclusions and future work are discussed in Section 4.

\section{Simulation Methodology and Workflow}

In this section, we will present a FEA simulation methodology and workflow on bonded repair of an aircraft structure experiencing an initial crack. The simulation procedure is carried out using Ansys suites functions. But the workflow is suitable and can be easily adopted using any existing FEA simulation software.

\subsection{FEA solution process for bonded repair simulation workflow (BRSW)}

Our proposed bonded repair effectiveness evaluation methodology is carried out in the following steps:

Aircraft structure geometry, crack geometry, repair patch geometry preparations. In this step, a CAD drawing is first completed based on the aircraft structure geometry and the initial crack geometry. The repair patch geometry is dependent on the repair strategy to be examined, e.g., stop-drill repair and damageremoval repair. An example of the geometry is shown in Figure 1.

1. Material properties preparation and assignments. In this step, the material properties are assigned for the aircraft structure to be repaired and the patch materials, e.g., boron patch and titanium patch. These material properties are necessary for the evaluation of crack growth and estimations of service life.

2. Aircraft structure and patch meshing generation, crack meshing generation. The meshing of the geometry is necessary for FEA simulation and analysis.

3. Aircraft structure loading and boundary conditions setup. This step sets up the loading and boundary conditions. In the case of fatigue tests, the loading is applied as cyclic loading.

4. Fatigue damage quantification. This step is to evaluate the damage using stress measurements caused by fatigue loading and to assess when crack is initiated.

5. Fatigue crack propagation simulation and service life quantification. This step is to provide an estimated service life for each type of the crack repair treatment.

The steps listed above is implemented through our proposed Bonded Repair Simulation Workflow (BRSW) with setup stages, shown in Figure 2. The BRSW process consists of three stages with building blocks which utilizes various Ansys software tools to perform designated tasks. The workflow starts from the definition of material properties (structures, patches, adhesives), to the problem setup (geometry preparation, mesh generation, loading and boundary conditions), to FEA solutions that yield fatigue damage evaluation, crack propagation quantification and service life estimation. 


\section{Hosted file}

image2.emf available at https://authorea.com/users/349641/articles/474610-evaluatingeffectiveness-in-bonded-repair-techniques-for-aircraft-structures-using-finite-elementsimulations

Stage 1 : The Material Preparation task utilizes Ansys Engineering Data tool [20] to prepare necessary material properties such as stiffness, strength, fatigue S-N properties, Paris Law, etc. The Geometry Preparation task utilizes Ansys SpaceClaim geometry tool [20] to import/create wing plank, crack, and repair patch geometries;

Stage 2 : During Mesh Generation task high quality finite element mesh is generated on prepared wing plank and repair patch geometries in Ansys Mesher tool [18], as well as to produce crack mesh. The Model Setup task is done through Ansys Mechanical tool [18], in which the practical loads and constrains are applied on wing plank structure, connecting repair patch, etc.;

Stage 3: The Fatigue Damage Analysis quantifies the fatigue damage accumulation in estimating crack initiation. It utilizes the Fatigue Tool capability in Ansys Mechanical to predict the repaired structure's extended service life before new crack initiates in repair techniques with crack tip treatments such as stopdrill or damage-removal. The Crack Propagation Analysis task will analyze the crack growth under fatigue loading, using the Fracture tool and SMART Crack Growth capabilities in Ansys Mechanical. The task produces the relationship between service loading cycles and crack advance quantity for unrepaired or repaired fatigue crack service life estimation.

\subsection{Aircraft damage tolerant repair methodology for different repair techniques}

To evaluate and compare the outcome of the repair techniques, we carry out the BRSW workflow described above for several scenarios, i.e., un-repaired, live-crack repair, and crack removal repairs, as shown in Figure 3 . The methodology starts with crack initiation estimation from either an undamaged wing structure or repaired wing structure with crack removal based on Fatigue Mechanics theory [21] and literatures [22], [23]. Fatigue crack propagation prediction with and without repairs on damaged wing structures employs Fracture Mechanics tools in Ansys software, where fracture parameters such as stress intensity factors are evaluated through SMART [19] and XFEM [18] FEA technologies, which in turn drive and quantify fatigue crack growth. The obtained relationship between crack growth and load cycles allows the service life estimation and repair decision guidance. As the damaged structure takes more service cycles leading to further fatigue damage, the FEA process automatically advances the crack growth and updates the further weakened structure integrity, accurately capturing the complex cohesion between damage state and remaining service life.

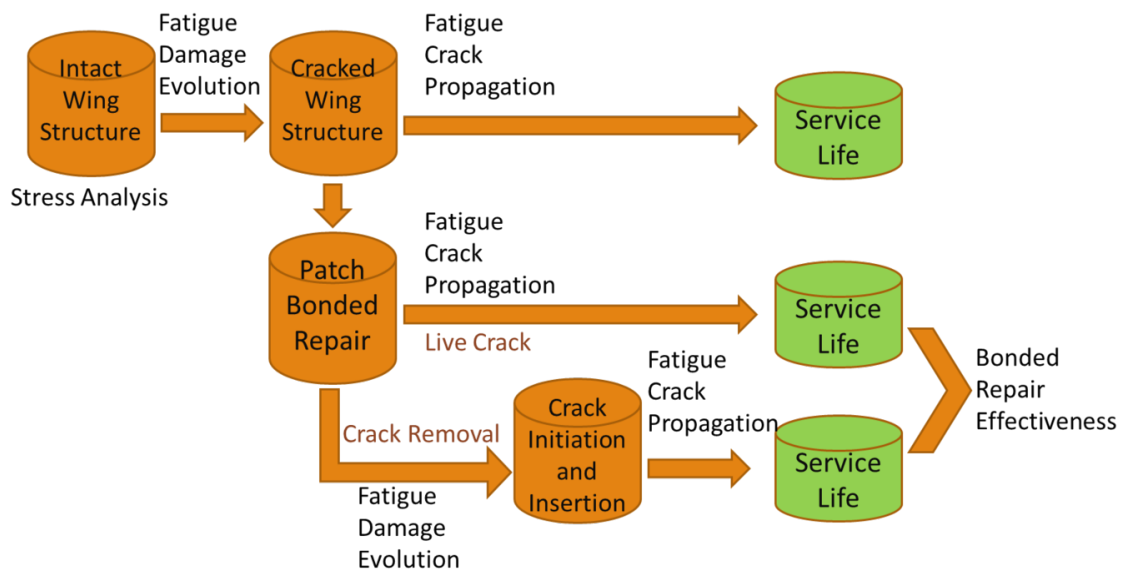


The first scenario presented in Figure 3 is the un-repaired service life. In this scenario, there is no applied patches. The loading on the wing structure is continuously applied. Crack is initiated based on the fatigue damage. BRSW process begins once the wing structure is damaged with a crack; the loading continues to evaluate the fatigue crack propagation. Finally, the service life is estimated based on the crack growth until failure.

The second scenario presented in Figure 3 is the live crack repair. In this scenario, once the aircraft structure is damaged with a crack from fatigue, a bolted repair patch is directly applied to the crack (shown in Figure 4a). Upon the application of the patch, the crack propagation is estimated with future fatigue loading. Finally, the service life is estimated for the bonded repair of a live crack using a patch.

The third scenario presented in Figure 3 is the crack removal repair. In this scenario, prior to a patch bonded repair as in the second scenario, the damage is physically removed. The crack removal can be performed in the forms of stop-drill (Figure 4b) or damage-removal (Figure 4c). In the stop-drill crack repair, two holes are drilled at both ends of the crack. In the damage-removal crack repair, the crack itself is removed. For each of these forms of the repair, cracks/damage are re-initiated. Crack propagation is continued to be assessed and their service lives are estimated due to fatigue loading.

\section{Hosted file}

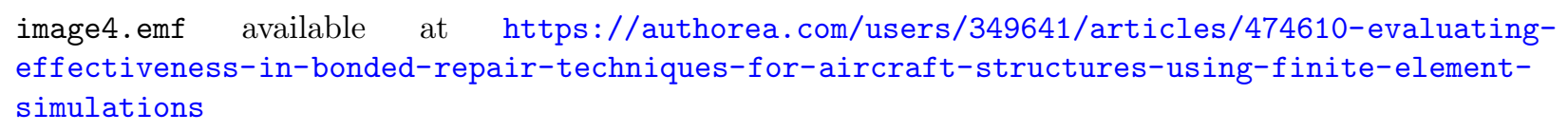

\section{Results and Discussions for Case Studies}

In this study, we will evaluate and compare the effectiveness of three aerostructure repair techniques using the simulation framework BRSW proposed in Section 2. Specifically, the comparisons are pertinent to prediction of fatigue damage evolution, crack initiation and propagation with service life estimation. The geometry under consideration is a cracked aircraft pressured fuselage repair. The setup and conditions are the same as in Ref. [20]. Without losing generality, the crack in damaged skin structure is placed in an arbitrary angle, as shown in Figure 5, even though cracks usually develop along rivet lines.

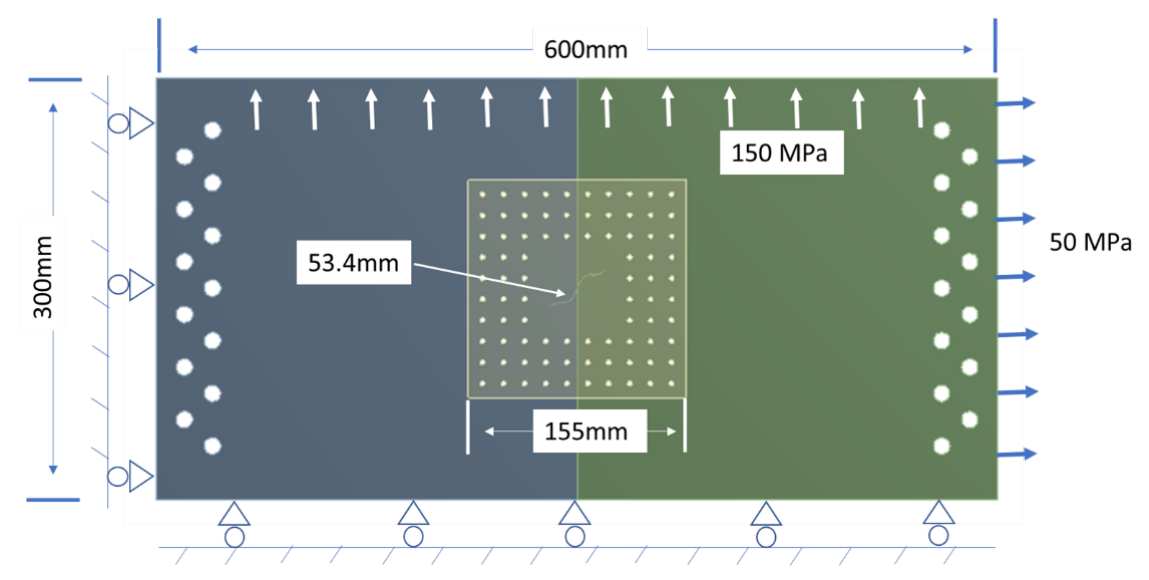

Figure 5 BRSW simulation on cracked aircraft pressured fuselage repair with repair patch in 3D analysis.

The damaged fuselage skin modeled is 600 by $300 \mathrm{~mm}$ and $1 \mathrm{~mm}$ thick, with a center crack of about $55 \mathrm{~mm}$ long. The skin material is $\mathrm{Al} 2024-\mathrm{T} 3$. The titanium repair patch is 155 by $155 \mathrm{~mm}^{2}$ andbolted onto the damaged structure skin (Figure 1). A typical pressurized fuselage loading scenario is applied with 150MPa pressure along peripheral direction and 50MPa in longitudinal direction. In this case study, Titanium alloy 
was used for the repair patch material and the patch is $1 \mathrm{~mm}$ thick. Here, four cases are examined; among them three crack treatments are considered in bolted patch repair:

1. Un-repaired cracked structure where there is no crack treatment is performed at all (fatigue crack propagation analysis is conducted in BRSW)

2. Live crack repair where patch bonded crack treatment (Titanium patch) is performed (fatigue crack propagation analysis is conducted in BRSW)

3. Stop-drill crack repair where crack front is drilled w/ stop hole in addition to Titanium patch bonded repair (fatigue damage analysis followed by crack propagation analysis in BRSW)

4. Damage-removal crack repair where crack region is cut out in addition to Titanium patch bonded repair (fatigue damage analysis followed by crack propagation analysis in BRSW)

For comparison, the fatigue crack growth and service life estimation on cracked skin for the un-repaired case is first analyzed as a reference. A thru-the-thickness crack of size $53.4 \mathrm{~mm}$ is initially inserted on the wing structure, as shown in Figure 6a. Figure 6b shows the fatigue crack growth of 5.3mm after 1027 service cycles, Figure 6c shows the crack growth of $49.1 \mathrm{~mm}$, reaching a total crack length of $102.5 \mathrm{~mm}$ after 4064 service cycles where it is considered structural failure [20] and the skin to be replaced. Figure $6 \mathrm{~d}$ shows the service life estimation in the relationship between fatigue crack growth and continued loading cycles. It is evident that BRSW FEA process is able to predict the fatigue crack growing direction deviating from its initial pre-crack orientation, driven by the practical service loading.

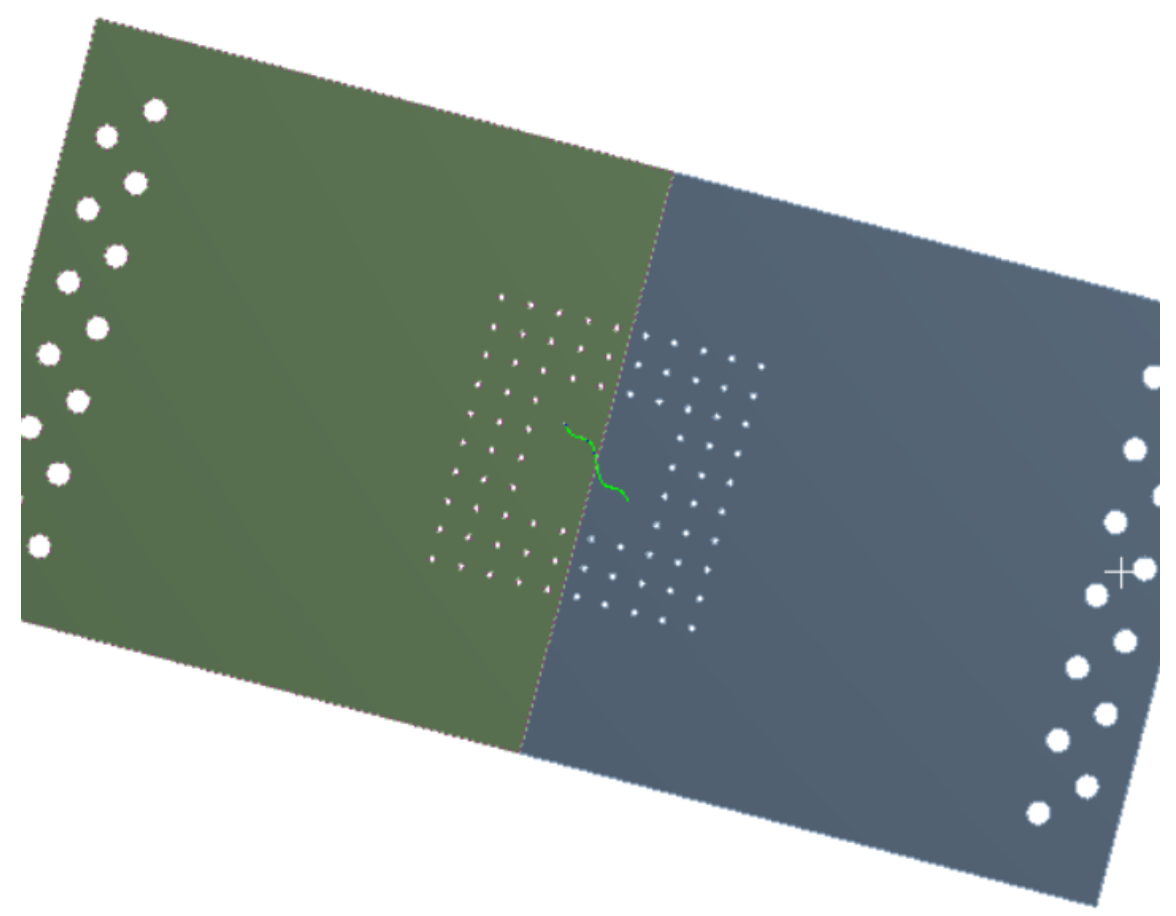

To illustrate the effectiveness of different repair techniques, the existing crack is treated using three ways using simulations: a) live crack repair (patch without crack treatment), b) stop-drill crack repair, and c) damage-removal repair (crack cutout), as shown in Figure 4. For each of the repair techniques, on the fatigue damaged fuselage skin structure with pre-crack, a bonded patch is placed.

For live crack repair, a patch is applied without any crack treatment, the fatigue loading continues to be applied until structural failure. The crack grows to $130.1 \mathrm{~mm}$ from its initial $53.4 \mathrm{~mm}$ initial crack. It is noted the two crack fronts grow additional $44.6 \mathrm{~mm}$ and $32.1 \mathrm{~mm}$. The fatigue life for live crack repair is extended from 4064 service cycles if no repair at all to 7598 service cycles, extending the wing structural life 
by nearly 2 -fold. As crack propagates, repair patch carries more and more loads leading to higher stresses as shown in Figure 7. Figure 7a shows the stress contours of the damaged structure with the crack and the titanium patch at 4249 loading cycles. Figure $7 \mathrm{~b}$ shows the stress of contours at 7598 cycles (at the end of the structural life). It is evident that the stress concentrates at the end of the crack tips and the crack continues to grow as more loading is applied. The stress on the titanium patch also continues to increase as more loading is applied and the crack grows.

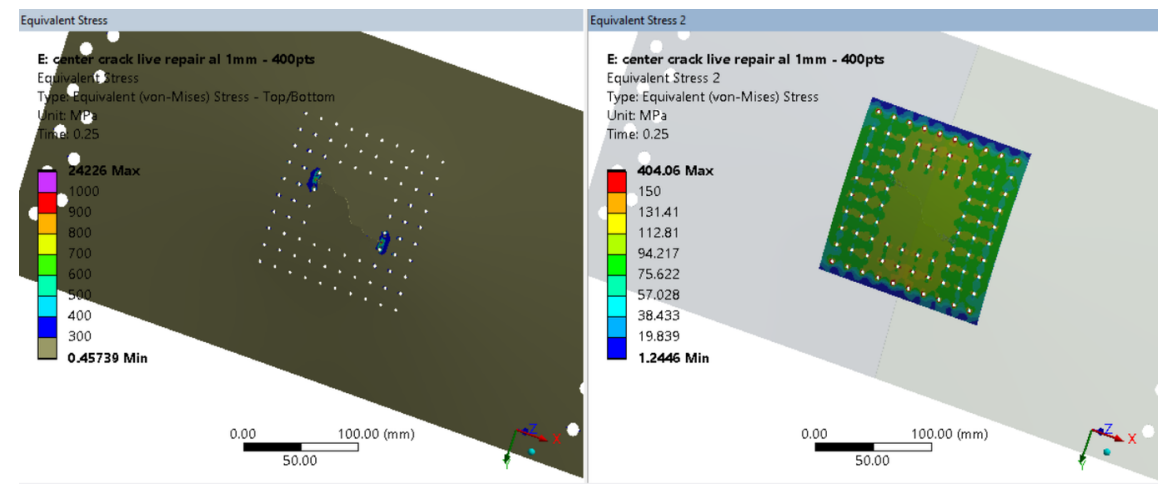

(a) Fatigue crack propagation (left) and titanium patch stress (right) at 4249 loading cycle

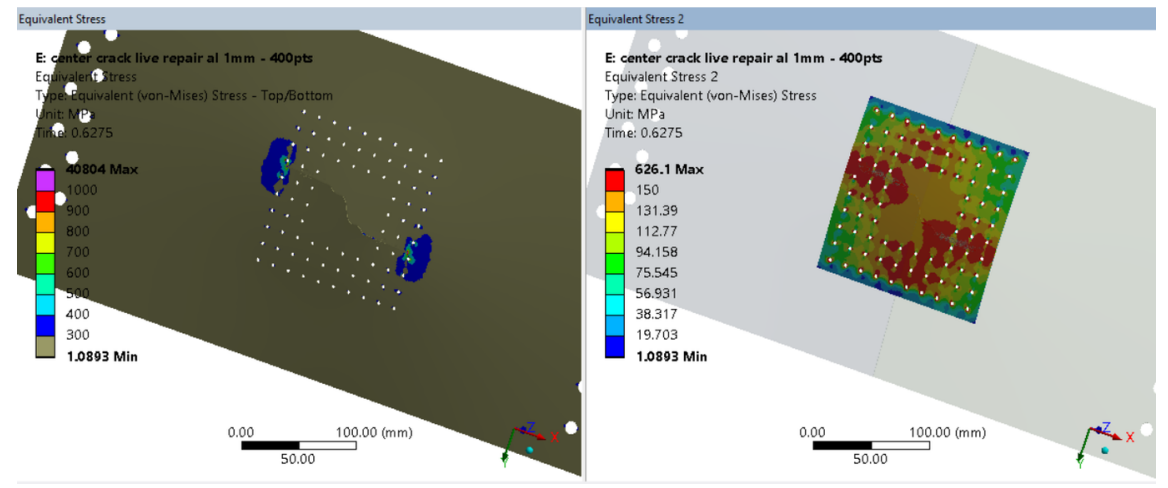

(b) Fatigue crack propagation (left) and titanium patch stress (right) at 7598 loading cycle

Figure 7 Stress distributions on the damaged structure and patch of a live repair.

For stop-drill crack repair, upon the initial crack appears, two holes of $3 \mathrm{~mm}$ in radius are drilled at each end of the crack to stop them from further growth. A patch is then placed. The fatigue loading continues to be applied. A re-initiation of a new crack of $1 \mathrm{~mm}$ occurs at 15,134 service cycles with stop-drill repair [23]. The fatigue crack propagation analysis is further performed. The crack ultimately reaches $76.9 \mathrm{~mm}$ with 27574 service cycles with stop-drill repair. The stop-drill repair technique extends the wing structural life by 6 -fold compared to no repair, and 3 times more effective compared to live crack repair. Figures 8a and 8b show the stress contours of fatigue crack propagation following its re-initiation at different load cycles. Figure 8a shows the stress concentration near the crack tips after re-initiation of the new crack upon stop-drill repair. The titanium patch exerts stress mostly concentrated in the general vicinity of the crack. Figure 8b shows the stress concentration of the crack tip at the end of its structural life, where the crack has grown to the end of the patched area. The patch stress increases correspondingly as more loading is applied and yields a more concentrated stress where the crack is located. 


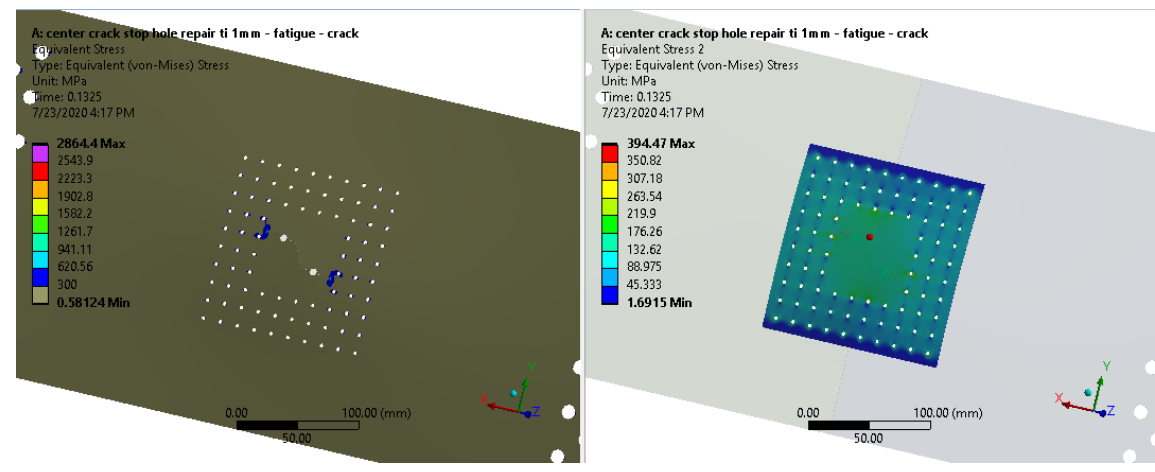

(a) Fatigue crack propagation (left) and titanium patch stress (right) at 19159 loading cycle

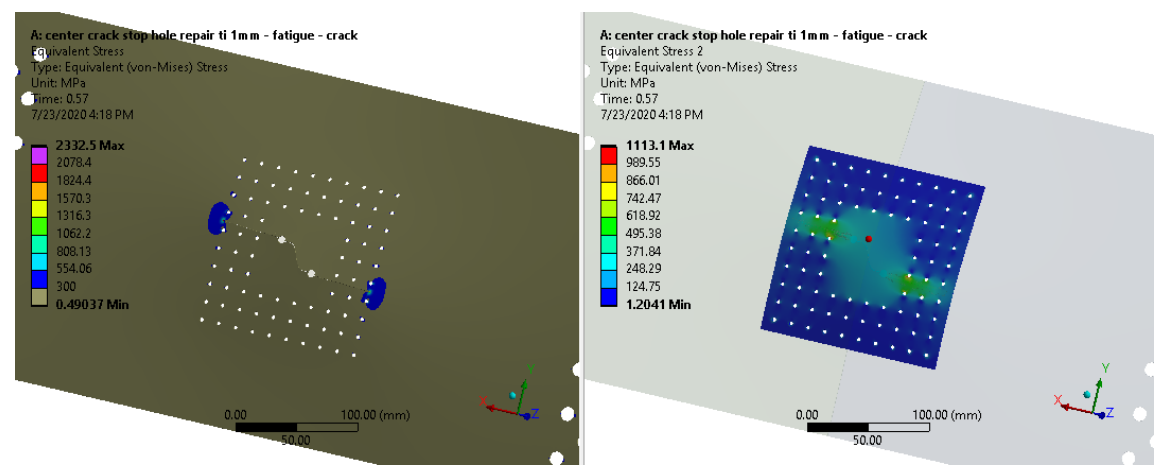

(b) Fatigue crack propagation (left) and titanium patch stress (right) at 27574 loading cycle

Figure 8 Stress distributions on the damaged structure and patch of a stop-drill repair.

Finally, for damage-removal repair, upon the initial crack appears, the entire damage cracked area is removed with a hole and a patch is placed. The fatigue loading continues to be applied. For damage-removal repair technique, the new crack occurs after 69,146 service cycles, a significant improvement compared to stop-drill repair (at 15,134 service cycles). The simulation continues to run until structural failure. Fatigue crack propagation analysis for further extended service life evaluation is then performed. The crack finally reaches $64.1 \mathrm{mmm}$ with 82645 service cycles. Damage-removal repair extends the wing structural life by 20 -fold compared to no-repair, 10 times more effective compared to live crack repair, and 3-times more effective compared to stop-drill repair. Figure 9 shows the von-Mises stress distributions of the damaged structure and the patch at different loading cycles. In Figure 9a, the crack starts to re-initiate at 70146 loading cycle and the stress concentrates begins to appear. The titanium patch has a relatively even stress distribution. At the end of the structural life (Figure 9b), the crack has propagated to the end of the patch region. Stress is concentrated at the end of the crack tip as well as the patch where the crack occurs. 

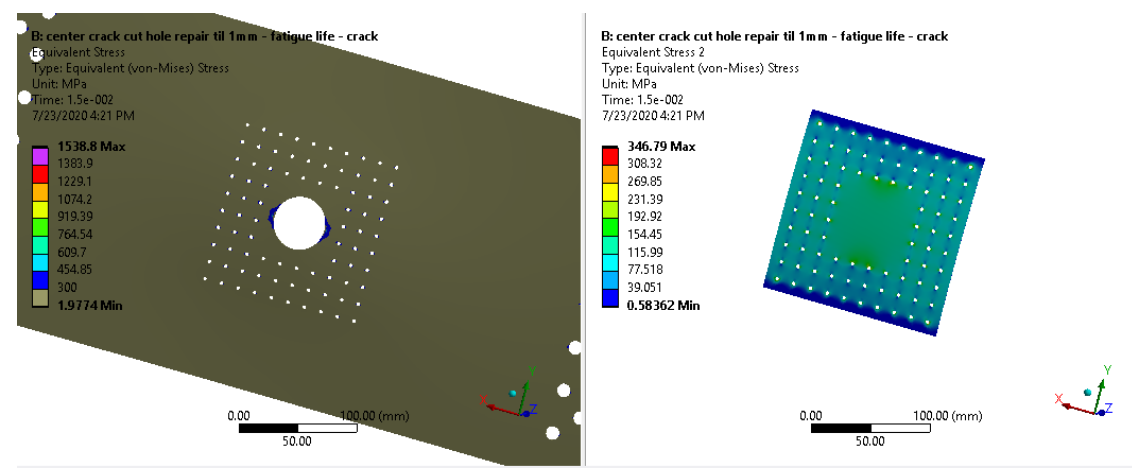

(a) Fatigue crack propagation (left) and titanium patch stress (right) at 70146 loading cycle
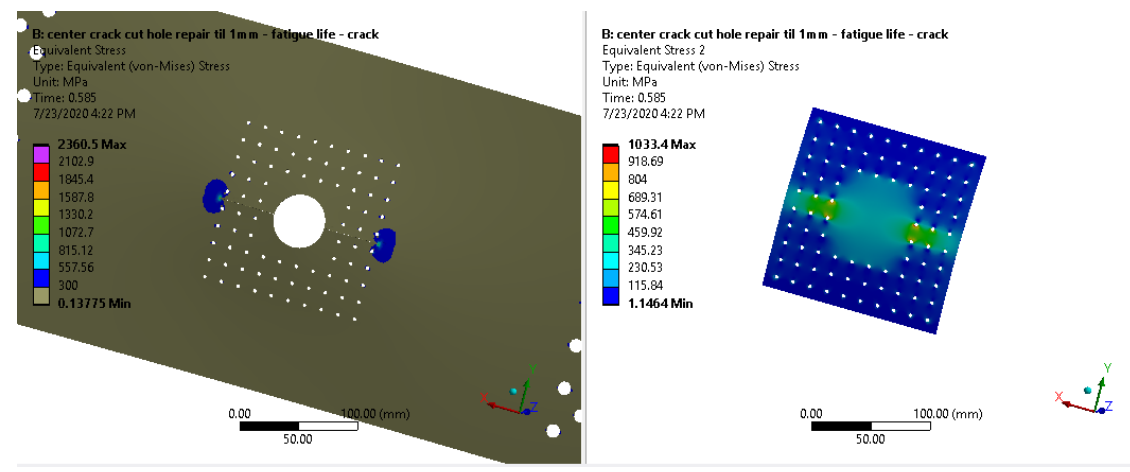

(b) Fatigue crack propagation (left) and titanium patch stress (right) at 82645 loading cycle

Figure 9 Stress distributions on the damaged structure and patch of a damage-removal repair.

A direct comparison of the effectiveness of each of the three repair techniques, namely live repair, stop-drill, and damage-removal repair, to un-repaired structure is summarized in Table 1 and shown in Figure 10.

Table 1 Service life comparison of different repair techniques.

\begin{tabular}{lll}
\hline Repair technique & Loading cycle at Crack re-initiation & Loading cycle at Service life \\
\hline No-repair & N/A & 4064 \\
Live-repair & N/A & 7598 \\
Stop-drill repair & 15134 & 27574 \\
Damage-removal repair & 69146 & 82645 \\
\hline
\end{tabular}

The result shows the enabled service life extension by performing a repair and their extended service lives for each technique. It is clear the load attraction by repair patch can greatly sustain fatigue crack growth, therefore leading to twice extended service life before the skin structure needs to be replaced, comparing to the live repair technique. By stop-drilling the pre-exist crack fronts, stop-drill repair is able to extend service life further by tolerating more fatigue damage in addition to sustaining new crack growth. Comparing to the small stop-drill hole, large damage-removal cutout further reduces stress concentration therefore slowing down fatigue damage accumulation and further extending service life. It is noted that not considering rivet holes could lead to overestimation on service life as fatigue damage is accumulated at those locations as well, leading to potential crack re-initiation. 


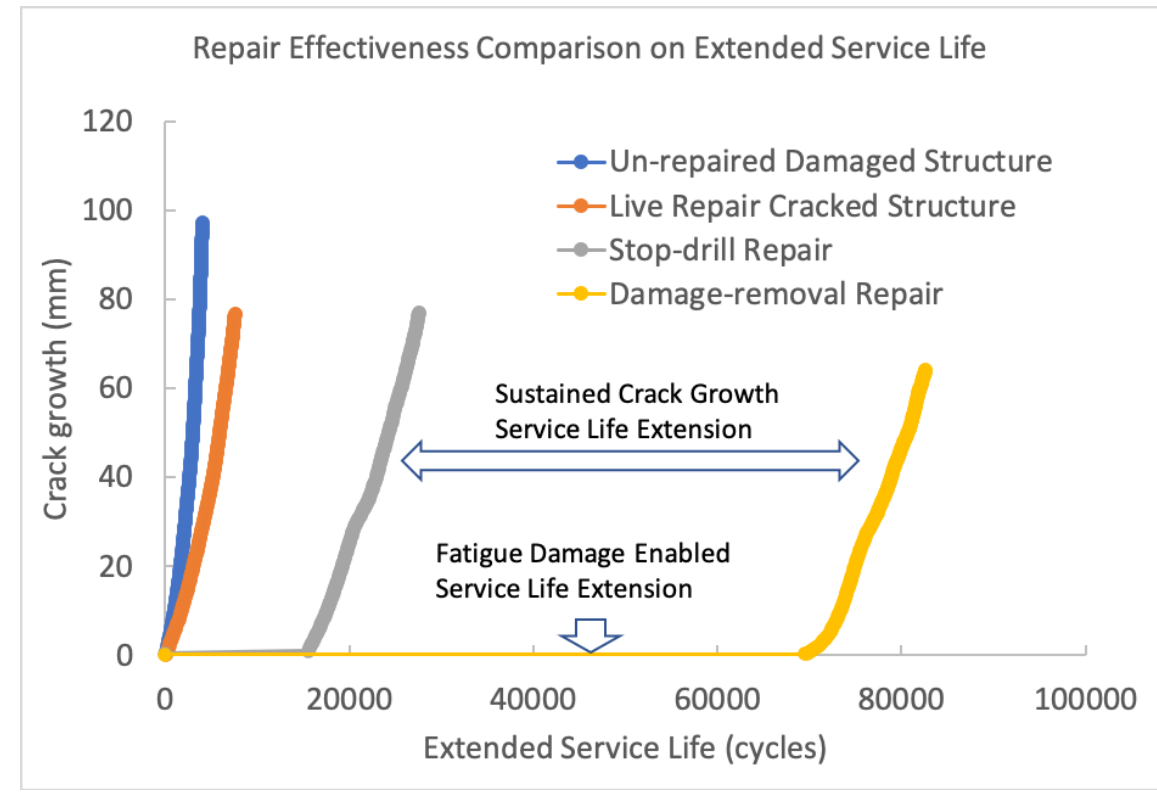

\section{Conclusions}

In this study, we developed and validated a FEA analysis process-Bonded Repair Structural Workflow (BRSW), from wing CAD model, cracks initiation and propagation under fatigue loading, to prediction and comparison of its service life before and after the bonded repairs. The simulations were performed using several of the built-in tools in Ansys software environment. Using the proposed BRSW workflow, we examined four case studies and compared the service life and sustained crack growth for each case. These four cases were un-repaired damaged structure, live repair, stop-drill repair, and damage-removal repair. The results showed that damage-removal repair yielded the best outcome by improving service life (in cycles) by at least three folds comparing to live repair and 20 times comparing to un-repaired. All three repair techniques are proven to be effective in reducing crack growth and extending service life compared to un-repaired case. Through the design of the workflow and the simulations of repair techniques, we successfully achieved the predictability of effectiveness of an aircraft structural repair. Traditional means of analytically predicting its service life with fatigue loading has limited the innovations of various repair techniques. We expect that with the proposed workflow, it is now easier and possible to investigate design new repair techniques.

Acknowledgement : This research did not receive any specific grant from funding agencies in the public, commercial, or not-for-profit sectors.

Data Availability: The processed data required to reproduce these findings are attached to data.mendeley.com for this submission.

\section{References}

[1] A. A. Baker and R. Jones, Eds., Bonded Repair of Aircraft Structures . Springer Netherlands, 1988.

[2] A. Vlot, S. Verhoeven, and P. J. M. Nijssen, Bonded Repairs For Aircraft Fuselages . 1998.

[3] C. Guijt and J. Mazza, "Case History: F-16 Feul Vent-hole Repairs," in Advances in the Bonded Composite Repair of Metallic Aircraft Structure, Elsevier Science Ltd., 2002.

[4] J. H. Svan, "Cracks found in Air National Guard C-130 wing parts prompt partial grounding of fleet," 2019. www.stripes.com.

[5] J. Paul, R. A. Bartholomeusz, and R. Jones, "Technical Note Bonded Load-Bearing Holes," Eng. Fract. Mech., vol. 48, no. 3, pp. 455-461, 1994. 
[6] Z. P. Marioli-Riga, G. J. Tsamasphyros, and G. N. Kanderakis, "Design of emergency aircraft repairs using composite patches," Mech. Compos. Mater. Struct., 2001, doi: 10.1080/107594101750370479.

[7] R. A. Bartholomeusz, J. J. Paul, and J. D. Roberts, "Application of bonded composite repair technology to civil aircraft. 747 Demonstrator program," 1991.

[8] C. H. Wang and C. N. Duong, Bonded Joints and Repairs to Composite Airframe Structures . 2015.

[9] K. Kaddouri, D. Ouinas, and B. Bachir Bouiadjra, "FE analysis of the behaviour of octagonal bonded composite repair in aircraft structures," Comput. Mater. Sci. , 2008, doi: 10.1016/j.commatsci.2008.03.003.

[10] NAVAIR, "Technical Manual, Engineering Manual Series, Aircraft and Missile Repair."

[11] NAVAIR, "Technical Manual, Cleaning and Corrosion Prevention and Control, Aerospace and NonAerospace Equipment."

[12] A. N. Rider, D. R. Arnott, and J. J. Mazza, "Chapter Seven - Surface Treatment and Repair Bonding," R. Jones, A. Baker, N. Matthews, and V. B. T.-A. S. and R. Champagne, Eds. Boston: ButterworthHeinemann, 2018, pp. 253-323.

[13] M. Caliskan, "Evaluation of bonded and bolted repair techniques with finite element method," Mater. Des. , vol. 27, no. 10, pp. 811-820, 2006, doi: https://doi.org/10.1016/j.matdes.2006.01.024.

[14] M. A. Caminero, S. Pavlopoulou, M. Lopez-Pedrosa, B. G. Nicolaisson, C. Pinna, and C. Soutis, "Analysis of adhesively bonded repairs in composites: Damage detection and prognosis," Compos. Struct. , 2013, doi: $10.1016 /$ j.compstruct.2012.07.028.

[15] R. Jones, D. Peng, and A. J. McMillan, "Chapter Five - Crack Growth From Naturally Occurring Material Discontinuities," R. Jones, A. Baker, N. Matthews, and V. B. T.-A. S. and R. Champagne, Eds. Boston: Butterworth-Heinemann, 2018, pp. 129-189.

[16] L. Gu, A. R. M. Kasavajhala, and S. Zhao, "Finite element analysis of cracks in aging aircraft structures with bonded composite-patch repairs," Compos. Part B Eng. , 2011, doi: 10.1016/j.compositesb.2010.11.014.

[17] R. Bartholomeusz, P. Pearce, and R. Vodicka, Advances in the Bonded Composite Repair of Metallic Aircraft Structure . 2002.

[18] Ansys Inc, "Mechanical Applications." 2019.

[19] Ansys Inc., "Mechanical APDL Theory Reference." 2019.

[20] R. Fredell, "Damage Tolerant Repair Techniques for Pressurized Aircraft Fuselages," Delft University of Technology, 1994.

[21] R. I. Stephens, A. Fatemi, R. R. Stephens, and H. O. Fuchs, Metal Fatigue in Engineering, 2nd Edition , 2nd ed. Wiley-Interscience, 2000.

[22] H. Grover, "Fatigue of Aircraft Structures," 1966.

[23] J. Schijve, "The Accumulation of Fatigue Damage in Aircraft Materials and Structures," Neuilly-SurSeine, 1972.

\section{Hosted file}

table1.doc available at https://authorea.com/users/349641/articles/474610-evaluatingeffectiveness-in-bonded-repair-techniques-for-aircraft-structures-using-finite-elementsimulations 

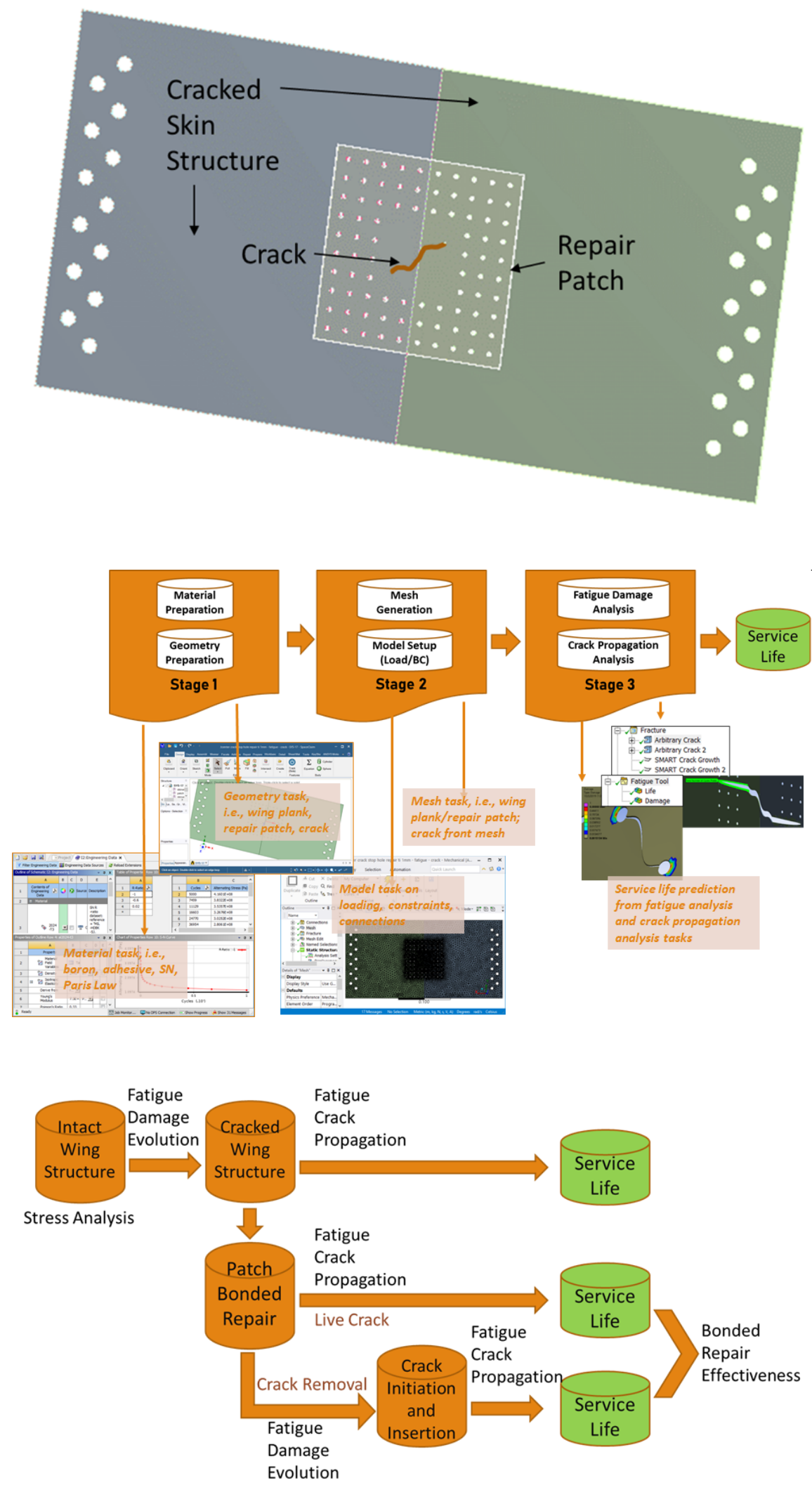

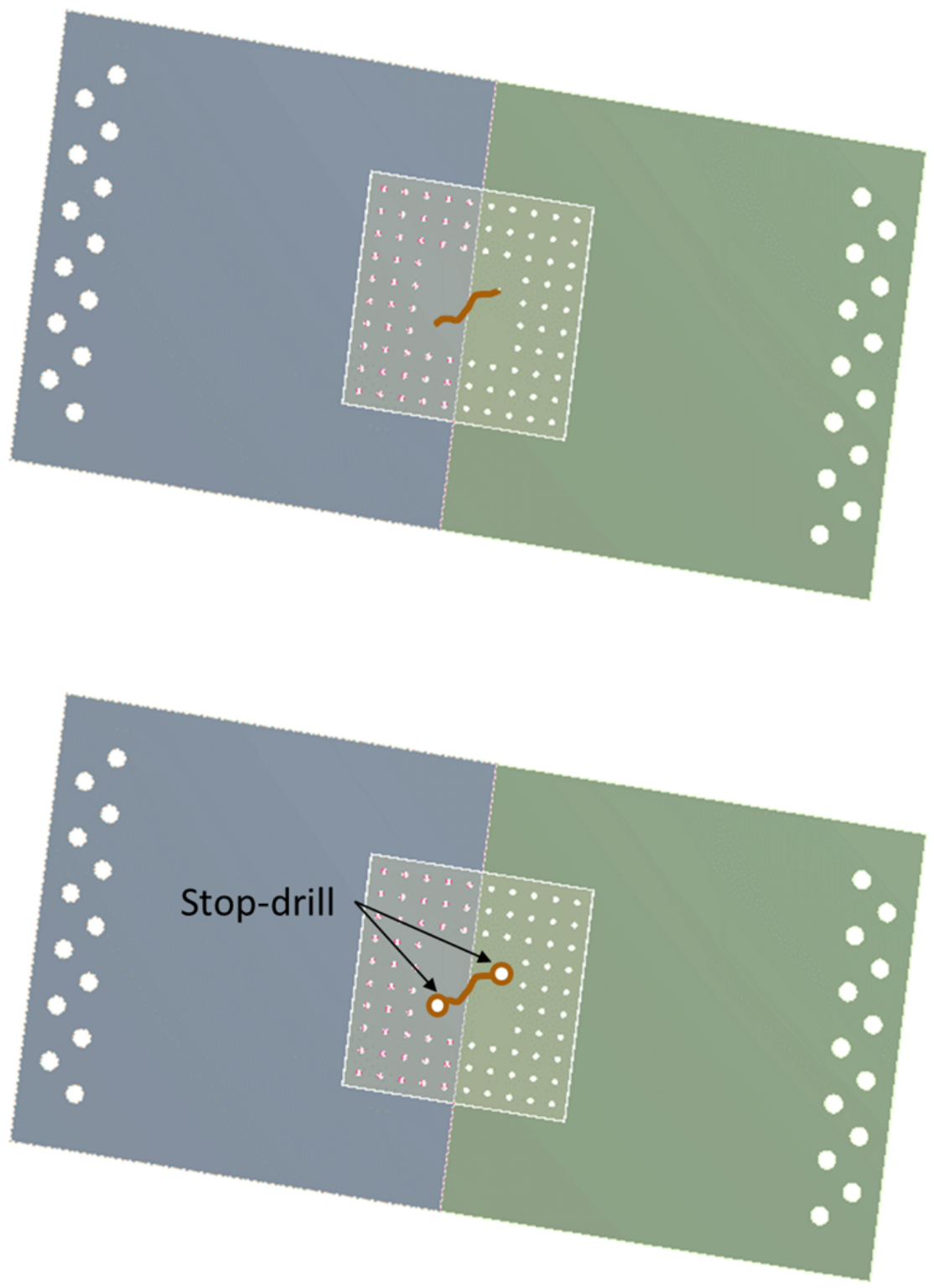

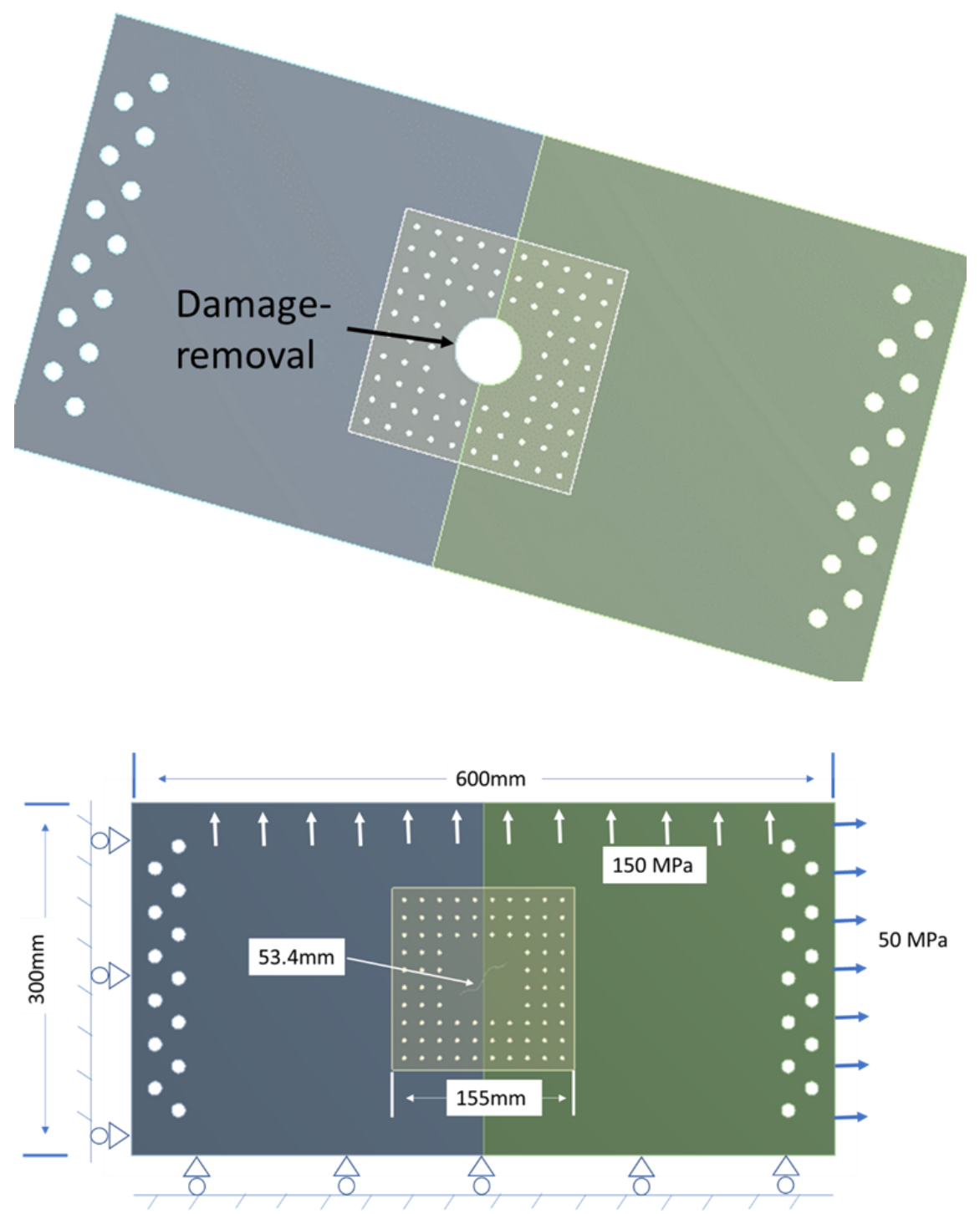

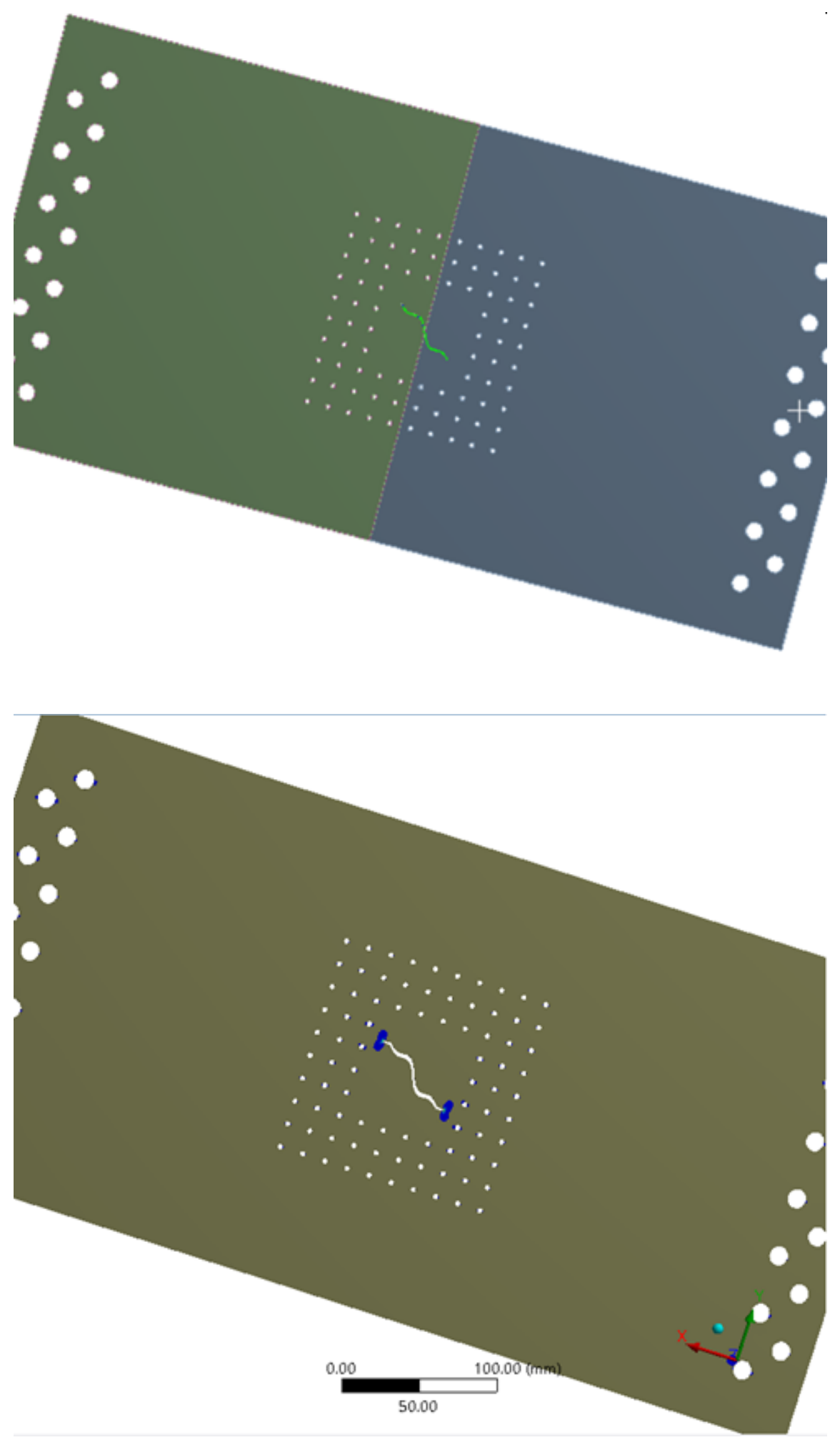

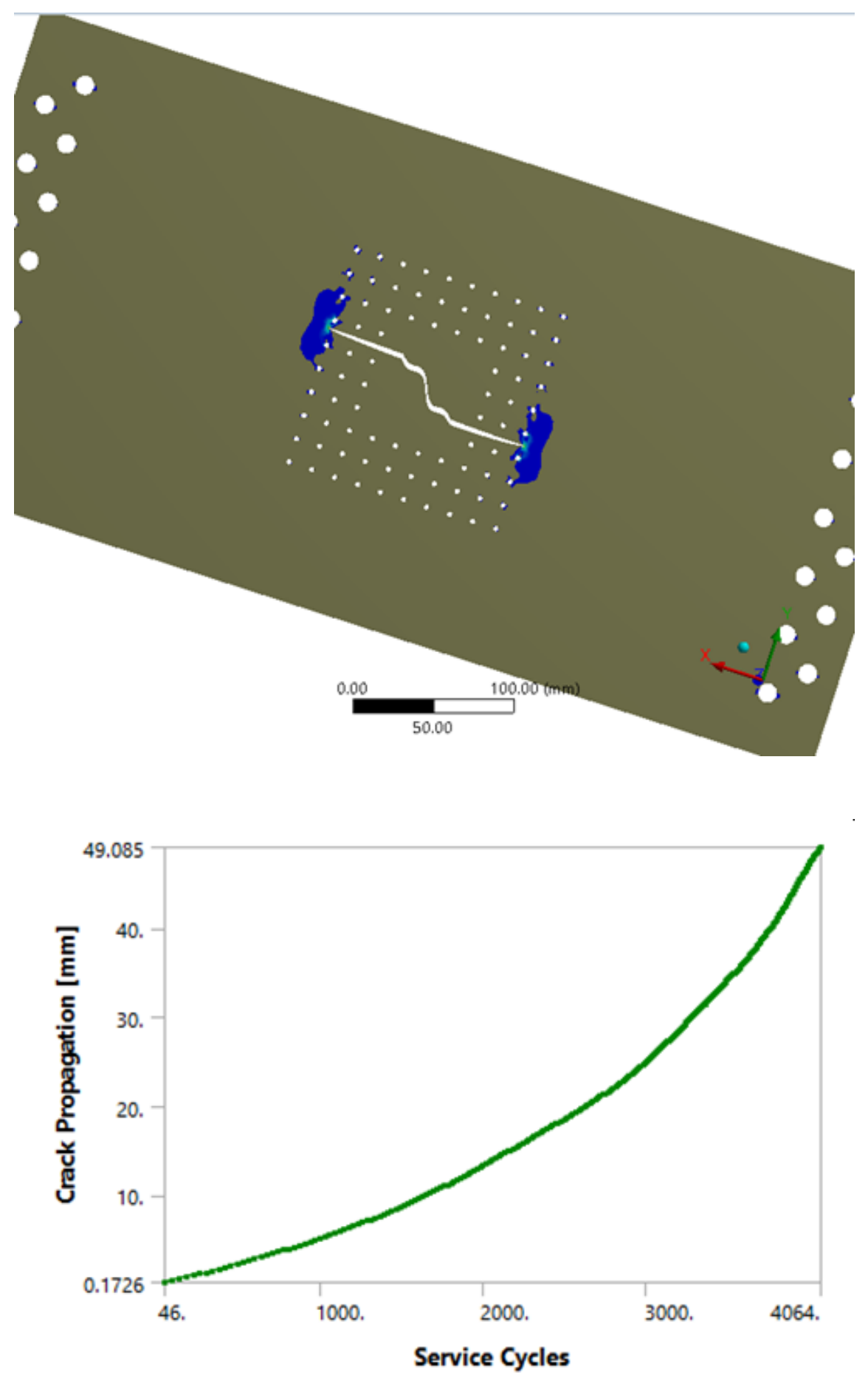

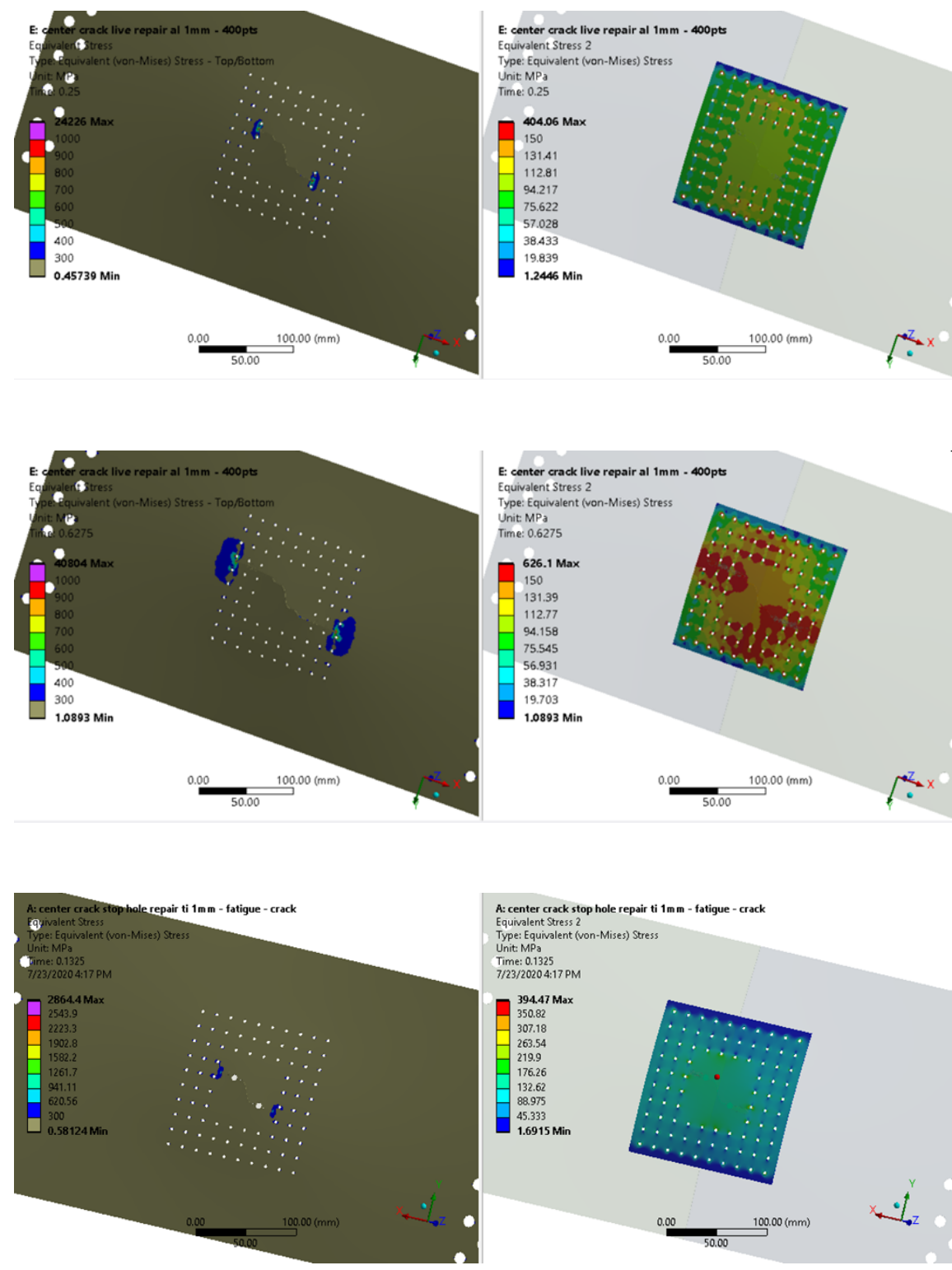

A: center crack stop hole repair ti $1 \mathrm{~mm}$ - fatigue - crack Equivalent Stress 2
Type: Equivalent (von-Mises) Stress
Unitt MPa Unit $\mathrm{MPa}$ a Time: 0.1325
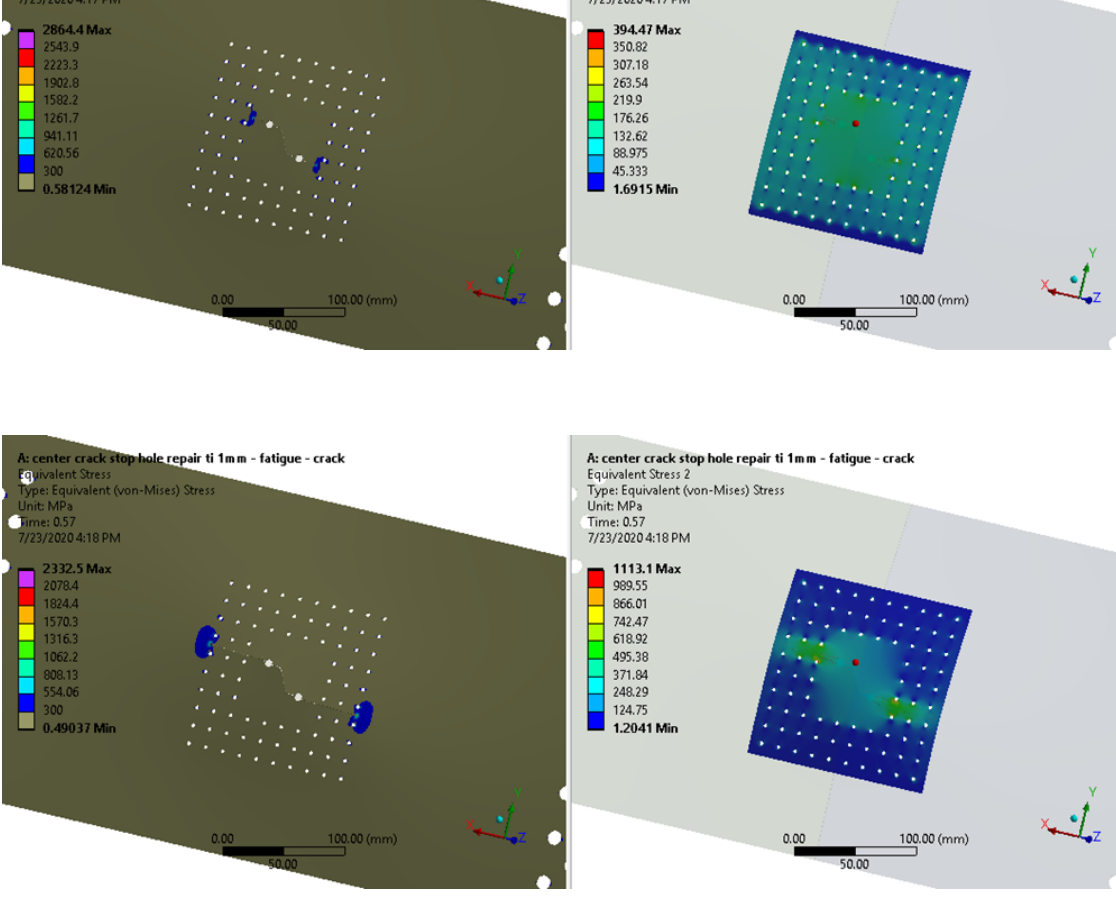

A: center crack stop hole repair ti $1 \mathrm{~mm} \mathrm{-} \mathrm{fatigue} \mathrm{-} \mathrm{crack}$ Equivalent Stress 2
Type: Equivalent (von-Mises) Stres Unit $\mathrm{MPa}$ 7/23/20204:18 PM

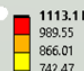

$\begin{array}{r}86601 \\ -742.47 \\ -618.92 \\ \hline\end{array}$

618.92
-495.38
371.84

37.24
248.29
124.75
1.2041 Min

1.2041 Min

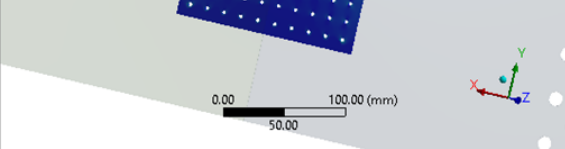



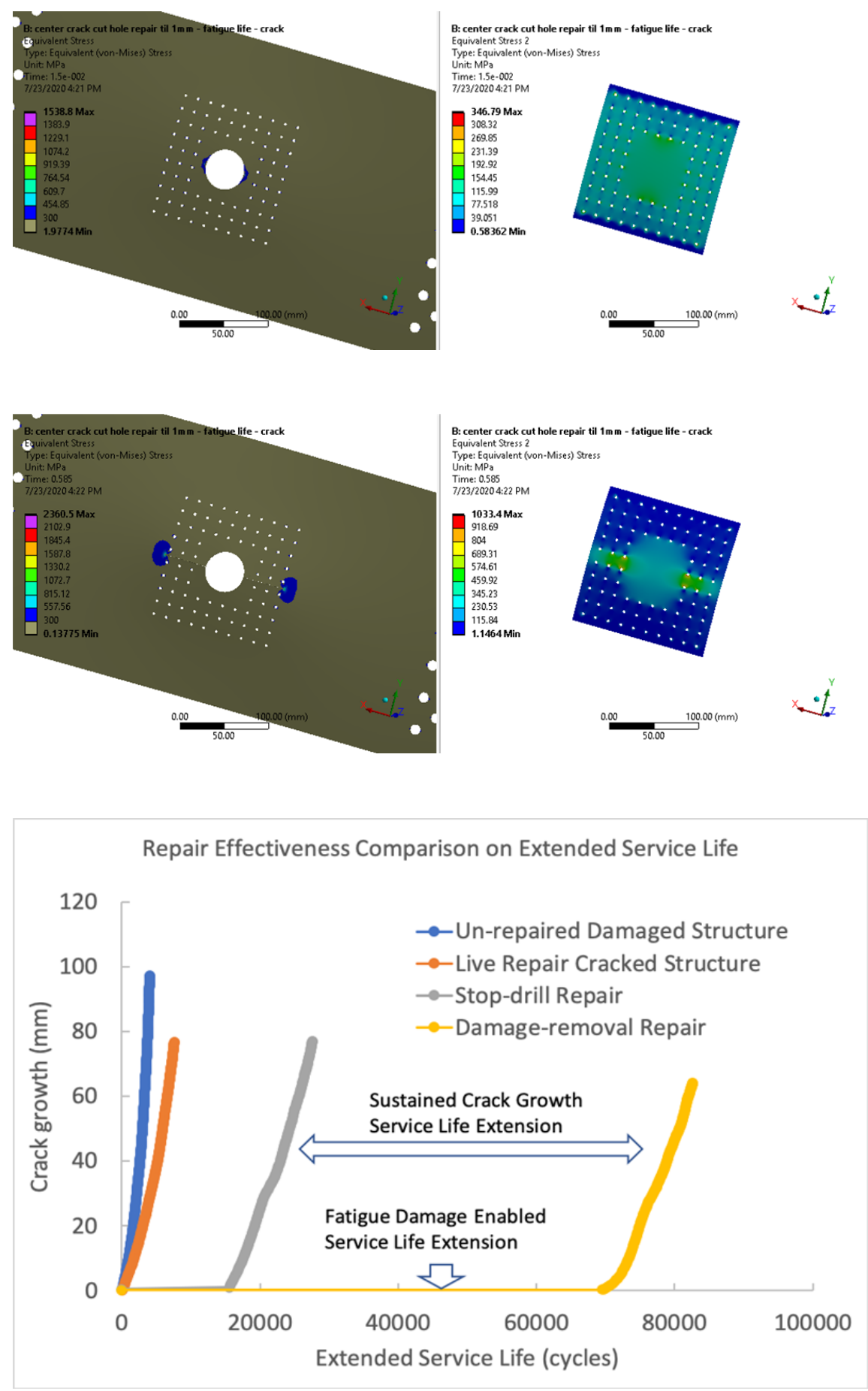